\title{
Disfunción eréctil: papel del laboratorio en la evaluación diagnóstica y pronóstica
}

\author{
J.V. García Cardoso, A. López Farré*, R. Vela Navarrete \\ Cátedra y Servicio de Urología. Fundación Jiménez Díaz. Universidad Autónoma. Madrid. \\ *Unidad de Investigación Cardiovascular. Hospital Clínico San Carlos. Universidad Complutense. Madrid.
}

Actas Urol Esp 2005; 29 (9): 890-898

\section{RESUMEN \\ DISFUNCIÓN ERÉCTIL: PAPEL DEL LABORATORIO EN LA EVALUACIÓN DIAGNÓSTICA Y PRONÓSTICA}

Objetivo: Revisar y actualizar las diferentes pruebas de laboratorio recomendadas para el diagnóstico etiológico de la disfunción eréctil y el efecto que pudiera tener sobre el pronóstico y tratamiento de la enfermedad.

Material y Métodos: Se han revisado las publicaciones más recientes y las que aportan nuevos conocimientos sobre los mecanismos etiopatogénicos y fisiopatológicos de la disfunción eréctil.

Resultados: La profundidad o extensión del protocolo analítico en la disfunción eréctil no ha de ser necesariamente la misma en todas las circunstancias. La edad, la coincidencia real o presumible de comorbilidades, marcan un límite diferencial entre los pacientes, exigiendo investigaciones complementarias que superan las demandas básicas.

Conclusión: La investigación etiopatogénica de la disfunción eréctil se ha ampliado considerablemente incluyendo nuevas pruebas de laboratorio. La tradicional búsqueda de comorbilidades como diabetes, disfunción hepática, hipogonadismo, hipercolesterolemia, se ha enriquecido con recientes valoraciones analíticas de potenciales marcadores de enfermedad endotelial.

Palabras clave: Disfunción eréctil. Diagnóstico de laboratorio. Disfunción endotelial.

\section{ABSTRACT \\ ERECTILE DYSFUNCTION: THE ROLE OF LABORATORY IN THE DIAGNOSTIC AND PRONOSTIC EVALUATION}

Objective: To review and to update the different laboratory tests recommended for etiologic diagnostic of erectile dysfunction and to evaluate the effect these tests could have on the pronostic and therapeutic strategy of this pathology.

Material and methods: We review the last articles related with etiopathogenics and pathophysiologics mechanisms of erectile dysfunction, including our studies on endothelial dysfunction and erectile dysfunction.

Results: The depth and extension of the laboratory protocol in erectile dysfunction is not necessaryly the same in all situations. The age, coincidence of comorbilities, set a different limit between patients demanding complementaries investigations that go beyond the basic request.

Conclusions: The etiopathogenic laboratory work up in erectile dysfunction is currently changing incorporating news tests. The traditional search of commorbilities like diabetes, hepatic dysfunction, hypogonadism, hyperglucemia is getting broad with recents analitics evaluations related with potential markers of endothelial disease. 
L os enfermos que habitualmente consultan por disfunción eréctil (DE) constituyen una población heterogénea por razones de edad, comorbilidades, gravedad de la DE y otros muchos motivos. En consecuencia, el acercamiento diagnóstico y pronóstico a estos enfermos tiene que ser necesariamente variado. McCullogh ${ }^{1}$ ha establecido una clasificación práctica tomando como referencia la edad, lo que permite considerar tres grupos de enfermos distintos: Los menores de 40 años; los mayores de 40 años y los mayores de 40 años con factores de riesgo conocidos. Desde esta perspectiva, que por si misma es útil porque los procesos coincidentes son más similares, el acercamiento al paciente impotente aporta una visión más realista y eficiente.

Los estudios de laboratorio deben acomodarse a cada paciente, basándose en los hallazgos de la historia clínica y la exploración física y deben de tratar de responder a las preguntas diagnósticas del clínico y tener repercusiones en el tratamiento. En la valoración de la disfunción eréctil la amplitud del estudio depende de la edad del paciente, de su estado de salud y de los fines que persigue el tratamiento. Más del 80\% de los casos de DE tienen un componente orgánico, y es habitualmente una manifestación más de una enfermedad sistémica, vascular, neurológica, endocrina o la coincidencia de todas ellas.

En la era en la que el manejo de los enfermos está íntimamente ligado a una relación costebeneficio no es admisible embarcar a los enfermos a una amplia batería de pruebas diagnósticas de laboratorio sin establecer unos criterios adecuados para cada paciente. La información obtenida de la anamnesis y del examen físico supone una guía indispensable para establecer las pruebas de laboratorio diagnósticas y los objetivos de un posible tratamiento. En este sentido, la Primera Consulta Internacional sobre Disfunción Eréctil ${ }^{2}$ ha establecido tres niveles de pruebas clínicas: recomendadas, opcionales y especializadas (Tabla 1). Las pruebas recomendadas son generalmente las que identifican o descartan un proceso patológico concreto, tal como diabetes, hiperlipemia o disfunción del eje hipotálamo-hipofisario. Las pruebas opcionales y especializadas requieren la contribución del especialista .
Tabla 1

Test de laboratorio para DE

\begin{tabular}{|c|c|c|}
\hline Recomendado & Opcional & Especializado \\
\hline Glucemia & Testosterona total & Vascular \\
\hline HbAlc & & Testosterona libre \\
\hline Perfil lipídico & Prolactina & Neurológico \\
\hline \multirow[t]{6}{*}{ Testosterona } & FSH & \\
\hline & SHBG & Psicogénico \\
\hline & Estradiol & \\
\hline & $\mathrm{TSH}$ & Hormonal \\
\hline & Urianálisis & \\
\hline & $\begin{array}{l}\text { Test psicológico } \\
\text { PSA }\end{array}$ & Estudio apnea \\
\hline
\end{tabular}

No existe consenso sobre el papel del laboratorio en los varones que presentan $\mathrm{DE}^{3-5}$. La glucemia en ayunas o una determinación de la HbAlc está indicada en los pacientes que tienen historia de poliuria o polidipsia a fin de evaluar la Diabetes Mellitus. Como la DE se asocia a hipercolesterolemia, un estudio del colesterol total con lipoproteinas de alta y baja densidad y de triglicéridos permitirá identificar el factor de riesgo arterioesclerótico de la DE. Los trastornos de la función tiroidea se manifiestan por síntomas muy diversos y, a todos los pacientes se les debería determinar la concentración de hormonas tiroideas. Como el hipogonadismo es una forma tratable de DE, habrá que determinar el valor de testosterona total en ayunas. Si éste valor es bajo debería de determinarse los valores de testosterona libre, LH y Prolactina para descartar un tumor hipofisario. Buvat y Lemaire ${ }^{6}$ revisaron 1.022 pacientes con disfunción eréctil y observaron que limitando la detección selectiva de los niveles de testosterona a los pacientes cuya exploración física era anormal, pasaban desapercibidos un 40\% de los casos con testosterona baja. Estos autores aconsejaron determinar la testosterona en todos los varones mayores de 50 años y en quienes se quejaban de escaso deseo sexual, menores de 50 años, con exploración clínica anormal.

Otro aspecto que afecta la estrategia diagnóstica y pronóstica en la DE es la nueva y confirmada consideración de que la $\mathrm{DE}$ frecuentemente no es una "enfermedad de órgano" sino la expresión de una enfermedad sistémica encuadrable en el 
grupo de las "disfunciones endoteliales. Esta consideración obliga al urólogo a indagar con más rigor y profundidad en la historia clínica del paciente en sus comorbilidades coincidentes o previsibles, especialmente las relacionadas con enfermedad cardiovascular (ECV), y establecer un marco analítico mucho más amplio de lo que hasta ahora era considerado como acercamiento básico, según las guías de comportamiento más generalizadas. De hecho puede afirmarse que el progreso científico en el campo de la DE ha supuesto un aumento de las exploraciones analíticas más que las instrumentales.

En definitiva, esta puesta al día intenta orientar al urólogo práctico sobre el profundo cambio de escenario producido en el diagnóstico, pronostico, prevención y tratamiento de la DE y el efecto que este progreso ha motivado sobre los estudios analíticos del paciente impotente.

\section{MATERIAL Y MÉTODOS}

Se han revisado las publicaciones más recientes que incorporan nuevos conocimientos sobre los mecanismos etiopatogénicos y fisiopatológicos de la DE modificando la demanda analítica básica y complementaria, con especial referencia a la consideración de la DE como "disfunción endotelial" (Tablas 2, 3 y 4). Se han comparado estos avances y requerimientos analíticos con las guías de comportamiento tradicionales de la AUA, EAU y OMS. Simultáneamente se ha hecho un análisis comparativo de la rentabilidad de estos mar-

Tabla 2

Mediadores de vasoconstricción en los cuerpos cavernosos

\begin{tabular}{ll}
\hline Noradrenalina & $\begin{array}{l}\text { Angiotensina } \\
\text { Endotelina 1 }\end{array}$ \\
$\begin{array}{l}\text { Super Oxidodismutasa (SOD) } \\
\text { Neuropeptido Y }\end{array}$ & \\
Prostanoides & Homocisteina \\
\hline
\end{tabular}

Tabla 3

Mediadores de relajación en los cuerpos cavernosos

Acetilcolina

Oxido nítrico

Polipéptido vasoactivo intestinal (VIP)

(Prostaciclina PGI2)

Peptido activador de la Adenil Ciclasa

Hipofisaria
Tabla 4

Potenciales marcadores de lesión endotelial activa.

Reactantes de fase aguda: Proteína $\mathrm{C}$ reactiva $(\mathrm{PCR})$ Neoptirina

BGF- $\beta 1$

Guanilil Cyclasa- $\beta 1$

EDIP

Colesterol total

Homocisteína

cadores diagnósticos y pronósticos, metabólicos, hormonales, de disfunción endotelial y otros en diferentes series cuantitativamente importantes de DE.

\section{RESULTADOS}

La profundidad o extensión del protocolo analítico en caso de disfunción eréctil no necesariamente ha de ser la misma en todas las circunstancias. Sin duda, la edad marca una diferencia importante entre estos grupos de población como señalado por McCullough ${ }^{1}$ y permite una orientación sobre el tema. Igualmente, la coincidencia real o presumible de comorbilidades como diabetes, obesidad, ECV, etc., marca también un limite diferencial entre los pacientes, exigiendo investigaciones complementarias que superan las demandas básicas. En cualquier caso, se han planteado diferentes controversias para la resolución objetiva del problema que han sido agrupadas, para su discusión, del siguiente modo: controversia sobre marcadores hormonales y DE; marcadores de disfunción del metabolismo lipídico; diabetes y DE y marcadores de disfunción endotelial.

\section{Marcadores hormonales y $D E$}

No hay ninguna duda actualmente de que la actividad sexual está regulada por influencias hormonales, tanto en su expresión funcional como por el deseo o libido. Seguramente hay umbrales de niveles androgénicos distintos que marcan diferencias funcionales importantes; por ejemplo niveles de castración abolirían tanto el deseo como la realización, aunque es conocido que estos niveles mínimos permiten erecciones en eunucos; los niveles fisiológicos serían necesarios, siempre relacionados con la edad, para mantener una función sexual "normal". El hipogonadismo del varón añoso, cuyas cifras 
nuevamente es preciso relacionar con la edad, motivarian deficiencias notables. Seguramente la controversia sobre el papel de los andrógenos en la DE está motivada no sólo por la existencia de "umbrales" precisos para comportamientos distintos, que necesariamente van ligados a la edad, sino en el acierto de elegir el marcador o los marcadores hormonales que mejor identifiquen la situación de deprivación o normalidad androgénica. En este sentido tenemos experiencia con testosterona total, testosterona libre, testosterona bioactiva, DHT, DHEA, DHEA-S, SGH, y otras hormonas. Se sigue indagando qué hormona o grupo de hormonas puede ser el mejor marcador en el caso de la DE, y en el caso del envejecimiento como tal, desde la perspectiva de que el envejecimiento incluye obligadamente una disfunción hormonal.

Bodie et al. ${ }^{3}$ indagaron en los datos de laboratorio de 3.547 pacientes con $\mathrm{DE}$ la prevalencia de anormalidades de laboratorio en los parámetros de testosterona (T), prolactina (Pr), LH, FSH, TSH, hemoglobina A1C, PSA, hemoglobina, colesterol y creatinina, encontrando que el $18,7 \%$ tenía una $\mathrm{T}$ baja, el 4,6\% incremento de $\mathrm{Pr}$, $14,6 \%$ valores anormales de $\mathrm{LH}, 4 \%$ aumento de TSH, 8,3\% aumento del PSA, 26,5\% anemia y $11,9 \%$ incremento de la creatinina. A la vez, hasta un $52,9 \%$ de estos pacientes tenía incremento de la $\mathrm{Hb}$ Alc y un $48,4 \%$ del colesterol. Este estudio, que confiere al laboratorio un relevante papel en el diagnóstico y pronostico de la $\mathrm{DE}$, contrasta con estudios previos en los que la incidencia de alteraciones de los marcadores hormonales fue muy baja. Concretamente, considerando anormalmente bajo un valor inferior a 220 $\mathrm{ng} / \mathrm{dl}$ de $\mathrm{T}$, se han señalado hallazgos variables entre $3,3 \% / 10,5 \%^{6,7}$. Se ha advertido que si se repitieran esos valores seguramente se encontrarían mayores deficiencias androgénicas en esta población de impotentes, y por supuesto el porcentaje se incrementaría de manera considerable si el umbral de normalidad se eleva a $280 \mathrm{ng} / \mathrm{dl}$. En conclusión Bodie et al. consideran que la deficiencia androgénica es común, es una condición tratable y la investigación rutinaria es razonable. No puede, en vista de su experiencia, aconsejar la determinación rutinaria de PR. Si aconseja la determinación de LH.

\section{Marcadores de disfunción lipídica}

Aunque la dislipemia es generalmente conocida como factor de riesgo de enfermedad cardiovascular y se ha asociado epidemiológicamente a la $\mathrm{DE}$, el valor de las pruebas de dislipemia en hombres con DE no ha sido suficientemente estudiado. Aproximadamente la mitad de los pacientes estudiados por Bodie $^{3}$ tenían valores anormales de colesterol. Korenman ${ }^{8}$ señala que hipercolesterolemia puede ser identificada hasta en un $70 \%$ de los varones con DE mayores de 60 años, Roumeguere ${ }^{9}$ encontró diferencias significativas en el colesterol total, HDL y ratio colesterol total/HDL. No identificaron estos parámetros como factores predictivos de DE, aunque si determinaron mayor riesgo de enfermedad coronaria. Por otra parte, se ha comprobado mejora de la DE tras el control de la hiperlipemia ${ }^{10}$. Recientemente Rao ${ }^{11}$ consideran a la HDL y ratio colesterol total/HDL como factores predictivos de DE. En conclusión, la alta prevalencia de aumento de la tasa de colesterol en esta población con DE justifica la determinación de colesterol total, con una ulterior determinación de las fracciones del mismo cuando encontrado elevado o en límites fisiológicos altos. La ratio colesterol total/ HDL y la fracción HDL son factores predictivos de DE.

\section{Glucemia y Hb glucoxilada}

La diabetes en un conocido factor de riesgo de enfermedad cardiovascular, arterioesclerótica, y de DE. Del 25 al 50\% de los pacientes que han sufrido diabetes tipo I por 25 años o más tienen $\mathrm{DE}^{12}$. Además, existen evidencias de que el control de la glucemia está relacionado con la gravedad de la DE en los individuos con diabetes tipo $\mathrm{II}^{13}$. Estos hallazgos justifican la determinación no sólo de la glucemia basal sino de la HBA1C, sobre todo debido a la mayor especificidad de esta ultima determinación. Sospechada la existencia de diabetes, la determinación de la glucemia en ayunas o un test de tolerancia a la glucosa es recomendable ${ }^{14}$.

\section{Marcadores de disfunción endotelial}

La conexión frecuente de DE y enfermedad cardiovascular (ECV) motivada por la patología sistémica de "disfunción endotelial" justifica una 
mayor agresividad diagnóstica en la $\mathrm{DE}$ en los casos sospechosos de ECV y una valoración de la gravedad del proceso en aquellos pacientes con manifiesta $\mathrm{ECV}^{15}$. Desgraciadamente, hasta el momento, no se conocen "marcadores de disfunción endotelial” realizados de manera sistemática en una población con DE. No obstante, hay varias líneas de investigación en este sentido, cuyos resultados más relevantes se mencionan a continuación.

Seong et al. ${ }^{16}$ han investigado el nivel plasmático de TGF/Beta 1 en un grupo de 62 pacientes con DE y comparado con 26 varones normales con edad similar. En el grupo de paciente con DE de origen vascular encontraron un aumento significativo $(6,7 \pm 4,9 \mathrm{ng} / \mathrm{ml})$ cuando comparado con el grupo control $(4,0 \pm 2,1 \mathrm{ng} / \mathrm{ml})(\mathrm{P}<0,01)$. Esto se refiere a toda la población con $\mathrm{DE}$, pero si comparamos con la población con $\mathrm{DE}$ vasculogénica el aumento fue más significativo ya que este grupo tenía 9,0 $\pm 5,5 \mathrm{ng} / \mathrm{ml}$. El TGF/Beta 1 produce fibrosis de los cuerpos cavernosos.

Las endotelinas tienen un papel importantísimo como reguladores a largo plazo del tono del músculo liso de los cuerpos cavernosos y un indudable papel en la fisiología y fisiopatología peneana. Sin embargo no han sido utilizadas como marcadores de disfunción endotelial. Las endotelinas modulan el efecto de otros agentes contráctiles como la noradrenalina, la proliferación celular y la expresión fenotípica. La detumescencia del músculo liso del cuerpo cavernoso se considera que está controlada por la liberación de varios neurotrasmisores y moduladores con efectos constrictivos, entre los son más importantes la noradrenalina y endotelinas. La inhibición de estos agonistas a nivel del receptor, o en la cascada de señalizaciones, debe conducir directamente a una disminución en la fosforolización reguladora de la miosina de cadenas ligeras y en consecuencia a una relajación mediante la reducción del calcio citoplasmático e inhibición de la sensibilación del calcio. Existen numerosos trabajos investigando el papel de cada uno de los marcadores mencionados en la tumescencia detumescencia peneana, pero ninguno de ellos ha sido utilizado como marcador de trastorno endotelial de manera especifica.
Aunque se conoce que muchos vasodilatadores endógenos tienen efecto relajante, tanto en las preparaciones aisladas de cuerpos cavernosos como de los vasos peneanos, parece evidente hoy día que la vía de relajación más importante de estas estructuras es la de L-arginina/ON/guanilciclasa/GMPc ${ }^{17}$. El papel prioritario del $\mathrm{ON}$ en la relajación del músculo liso cavernoso y de los vasos peneanos está actualmente fuera de toda duda. El origen de este ON puede provenir de los nervios de los cuerpos cavernosos o del endotelio, de tal modo que más de una isoforma de la ON sintasa (ONS) participa en el proceso; la ONSn o neuronal, y la ONSe o endotelial. Ambas son activadas por la entrada de calcio en la célula ligado a Calmodulina asociada con variadas enzimas. Este proceso es de corta duración, muy inferior a la duración de la erección peneana. La vía del ON sirve para caracterizar el grado de disfunción endotelial. Parece razonable indagar la vía del $\mathrm{ON}$ para detectar, si en caso de disfunción endotelial, existe alguna alteración significativa y repetible, que comparada con situaciones de normalidad fisiológica adviertan sobre la existencia de marcadores de disfunción endotelial aplicables en clínica. Este es el camino que han seguido los trabajos de nuestro grupo ${ }^{18}$, confirmando que la $\mathrm{DE}$ de causa vascular produce un considerable aumento de la Guanilato cyclasa $\beta 1$ (GC $\beta 1$ ) muy superior al producido de manera general en otros casos de DE.

¿Se podría reconocer en enfermos con disfunción eréctil la probabilidad de desarrollar enfermedad endotelial?

¿Podríamos disponer de un test diagnóstico de laboratorio marcador de enfermedad endotelial?

Recientemente, se ha evidenciado que la liberación de NO no neuronal en el cuerpo cavernoso es un aporte adicional proveniente del endotelio vascular y probablemente del músculo liso. Así, evidencias experimentales sugieren que la isoforma endotelial de la óxido nítrico sintasa (ONSe) y la subsiguiente producción de NO podría tener una importancia significativa en la relajación del cuerpo cavernoso al objetivar que ratones transgénicos carentes de ONSn tienen capacidad para tener actividad eréctil previa estimulación de los nervios pélvicos. 
Además, tinciones inmunohistoquímicas han demostrado la presencia de ONSe en el endotelio de los sinusoides cavernosos, en el músculo liso trabecular y en las paredes de las arterias helicinas ${ }^{19,20}$.

Los argumentos previos resaltan que, a pesar de la importancia de la localización neuronal de la ONS (ONSn), es necesario una función endotelial intacta para una función eréctil correcta abriéndose un nuevo concepto al considerar esta patología integrada dentro de un grupo de patologías en las que la alteración del endotelio es nexo común.

La disfunción eréctil se observa a menudo en varones con enfermedad cardiovascular, y algunos investigadores han sugerido que la disfunción eréctil puede ser un heraldo de este trastorno. En los estudios epidemiológicos, la hipertensión, la cardiopatía isquémica y la enfermedad vascular periférica se asocian con frecuencia a la disfunción eréctil. La asociación entre disfunción eréctil y enfermedad coronaria está respaldada por los resultados obtenidos en varios estudios que correlacionan la enfermedad coronaria con la dificultad para alcanzar erecciones ${ }^{21}$. En algunos casos, el inicio de la disfunción eréctil fue previo a los sintomas y al diagnóstico de enfermedad coronaria. Así las evidencias clínicas sugieren un nexo común fisiopatológico.

Recientemente, nuestro grupo describió cómo distintas citoquinas disminuyen la expresión de la proteína ONSe reduciendo la vida media del ARN mensajero de ONSe mediante la activación de la interacción de una proteína citosólica del endotelio con la región 3'-UTR del ARN mensajero de la NOSe por lo que esta proteína se definió como inductora de la disfunción endotelial $(\mathrm{EDIP})^{22,23}$.

Hemos estudiado la ruta metabólica del ON en las células mononucleares, sangre periférica y cuerpo cavernoso, obtenidas de pacientes con disfunción eréctil de origen vascular y neurológico.

Los resultados de nuestro trabajo demuestran que el sistema ON/GMPc está alterado, tanto a nivel sistémico como en el cuerpo cavernoso, existiendo una disminución de la ONSe y un aumento GCs y la EDIP. Las variaciones son estadísticamente significativas en los enfermos con disfunción eréctil de origen vascular (Figs. 1,2,3). Los estudios con microscopía confocal de las células mononucleares de estos enfermos confirman estos hallazgos (Fig. 4). Por lo tanto, en un futuro cabría la posibilidad de introducir la determinación de estas moléculas en la batería rutinaria de pruebas de laboratorio en este tipo de enfermos $^{24}$.

El papel de los marcadores de inflamación en el diagnóstico y pronóstico de enfermedad endotelial, especialmente ateroesclerótica, han sido revisados recientemente ${ }^{25}$. El mecanismo inflamatorio de la arterioesclerosis está abriendo vías

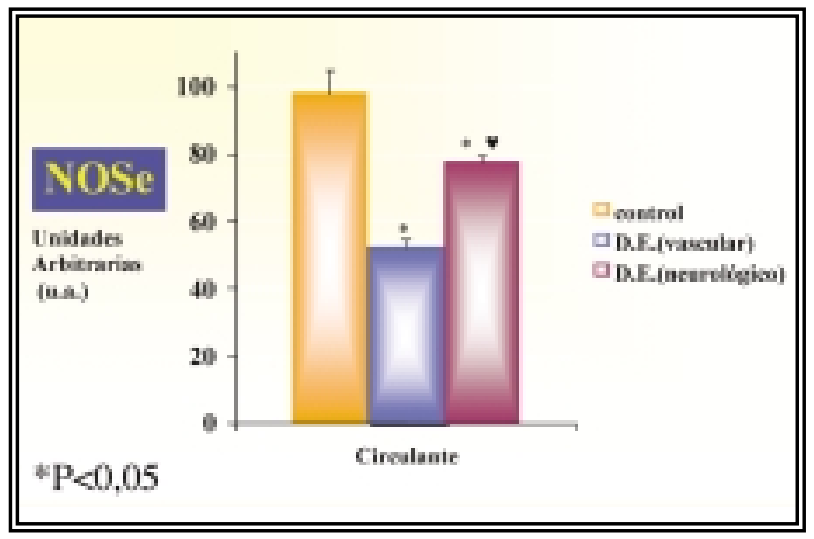

FIGURA 1. Diagrama de barras que representa la expresión de la NOSe en las células mononucleares de sangre circulante de los pacientes control y de los pacientes con $D E$ de origen vascular $y$ neurológico.

${ }^{*} p<0,05$ con respecto al control. $p<0.05$ con respecto a las células mononucleares de los pacientes con disfunción eréctil de origen vascular.

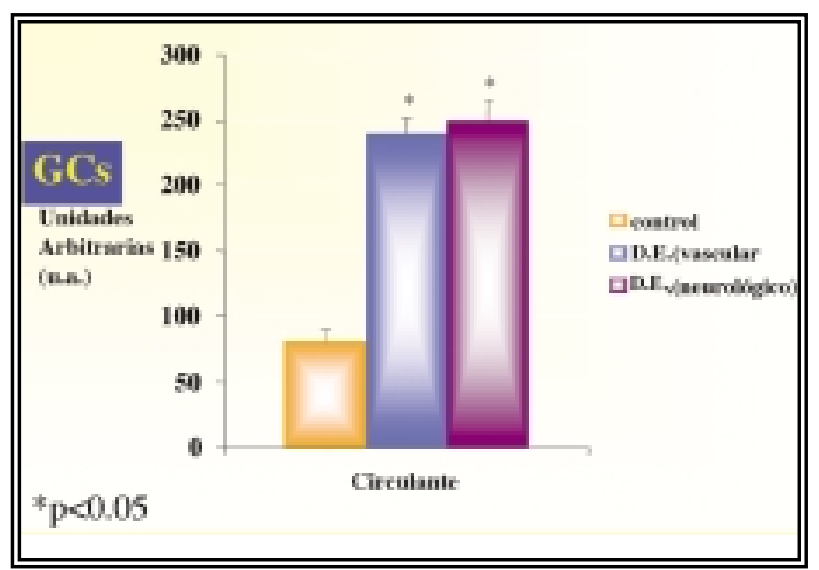

FIGURA 2: Diagrama de barras que representa la expresión de la GCs en las células mononucleares de sangre circulante de los pacientes control y de los pacientes con $D E$ de origen vascular y neurológico.

${ }^{*} p<0,05$ con respecto al control. 


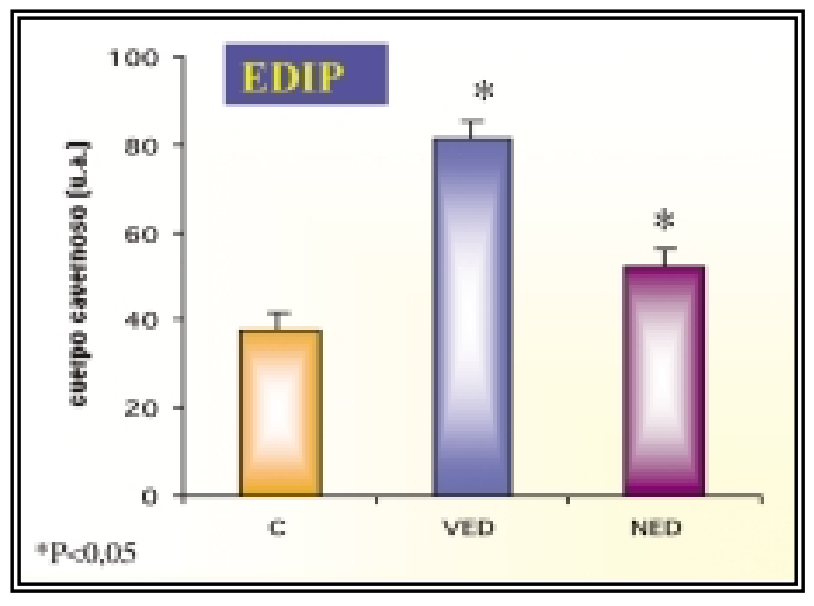

FIGURA 3: Diagrama de barras que representa la expresión de la proteina EDIP en las células mononucleares de sangre circulante de los pacientes control (C) y de los pacientes con DE de origen vascular (VED) y neurológico (VEN). ${ }^{*} p<0.05$ con respecto al control

\section{OXIDO NITRICO SINTASA ENDOTELIAL.}
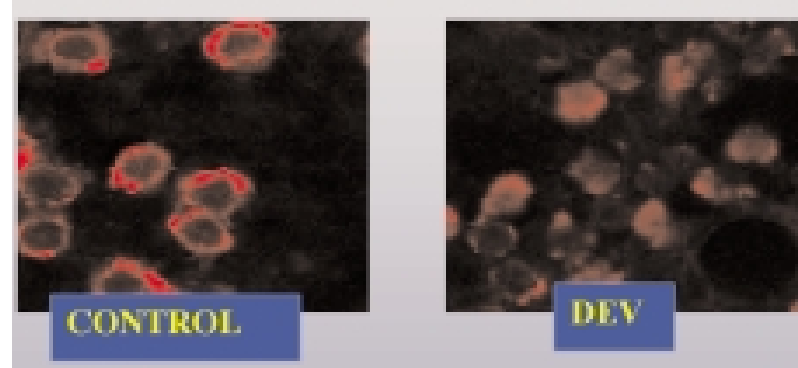

GUANILATO CICLASA SOLUBLE

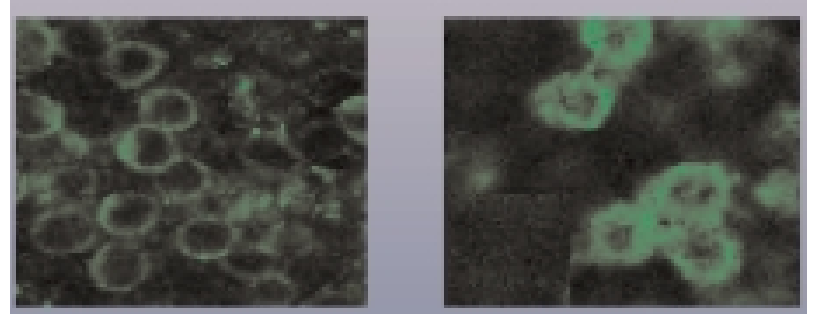

FIGURA 4: Imágenes de microscopia confocal mostrando la expresión de la subunidad bl de la Guanilato ciclasa soluble y de la NOSe en células mononucleares de pacientes sanos y de pacientes con disfunción eréctil de origen vascular (DEV). Se aprecia una mayor captación de la NOSe y una menor capatación de la subunidad b1 de la Guanilato ciclasa soluble en los casos control.

de investigación muy interesantes y tanto los reactantes de fase aguda como los de fase crónica están siendo considerados como potenciales marcadores diagnósticos que abran el camino de opciones preventivas. Entre los reactantes de fase aguda sobresale el esfuerzo dedicado a la proteina $\mathrm{C}$ reactiva (PCR). La PCR es predictora, independiente de mal pronóstico, durante el seguimiento a 4 años de enfermedad cardíaca (estudio Capture). Desde el punto de vista diagnóstico es importante señalar que los niveles referidos de PCR son de rangos bajos entre 1-10 $\mu \mathrm{g} / \mathrm{ml}$. Niveles superiores a $10 \mu \mathrm{g} / \mathrm{ml}$ son irrelevantes para la patología cardiovascular. Hay numerosos trabajos confirmativos de que la elevación de los niveles de PCR tienen valor pronóstico independiente en pacientes con enfermedad arterioesclerótica. Destacan los estudio MRFIT ${ }^{26}$, el PHS $^{27}$. En la mayoría de estos estudios señalan que niveles entre 1-40 y 2-40 son valores predictivos de riesgo vascular. Los niveles de PCR son significativamente mayores en obesos con DE.

También han sido valorados los reactantes de fase crónica y entre ellos el factor transformador del crecimiento- $\beta$ (TGF- $\beta$ ) y otras citoquinas proinflamatorias como IL-1, IL-6, factor $\alpha$ de necrosis tumoral (TNF $\alpha$ ). Varios estudios han demostrado la correlación entre niveles plasmáticos de citoquinas y arteroesclerosis de forma que se ha propuesto que la determinación de los niveles plasmáticos de citoquinas pueden ser utiles para pacientes con riesgo de sufrir eventos cardiovasculares $^{28,29}$. La neopterina, un derivado nepteridínico secretado por macrófagos tras estimulación por IFN $\gamma$, parece ser un buen marcador de arterioesclerosis en arterias periféricas y más recientemente de lesiones de cardiopatía isquémica ${ }^{30,31}$.

La velocidad de sedimentación y el recuento leucocitario han sido investigados también como expresión de un proceso inflamatorio, en fase aguda, para valoraciones pronósticas en caso de infarto de miocardio.

Por otra parte, estudios epidemiológicos, clíni$\cos$ y de laboratorio sugieren que las concentraciones elevadas de Homocisteina en plasma aceleran la enfermedad cardiovascular al favorecer la expresión de factores inflamatorios vasculares, la disfunción endotelial y la hipercoagulabilidad. Aunque la disminución de las cifras de esta molécula mediante la terapia con ácido fólico no ha evidenciado la reducción de los biomarcadores de la inflamación, no hay suficiente evidencias para recomendar su uso en la práctica clínica rutina$\mathrm{ria}^{32-34}$ 


\section{CONCLUSIÓN}

La investigación que proporcionan las pruebas convencionales de laboratorio no aportan, en muchos casos, la información necesaria para valorar el diagnóstico etiopatogénico el grado de severidad de la DE. Su principal misión debería de ser identificar factores de riesgo de $\mathrm{DE}$ que precisasen tratamiento específico para, así, ofrecer alternativas terapéuticas definitivas y no sólo un tratamiento sintomático. Los objetivos del protocolo analítico deberían determinar la presencia y severidad de enfermedad sistémica (vascular, neurológica o endocrina) e identificar pacientes susceptibles de respuesta a determinados tratamientos.

Nuestra revisión concluye que los niveles de testosterona, los parámetros de función hipofisaria, niveles de colesterol y HbAlc deberian de incluirse de forma programada. Los hallazgos de nuestro grupo, en la ruta metabólica del ON, aventuran que, en un futuro próximo, la adicción de marcadores de la función endotelial pueden tener implicaciones diagnósticas y pronósticas importantes.

\section{REFERENCIAS}

1. Mcculloch D, Campbell I, Wu F, Clarke B, Prescott. Impotence in diabetic and non-diabetic hospital outpatients. Br Med J 1980;281(6249): 1216.

2. Broderick G, LUE. In Campbell's Urology. Walsh PC, Retik $\mathrm{AB}$, Vaughan ED and Wein AJ (eds). Saunders Philadelphia. 2002.

3. Bodie J, Lewis J, Schow D, Monga M. Laboratory evaluations of erectile dysfunction: An evidence approach. J Urol 2003;169(6):2262-2264.

4. Mclaughlin SP, Carson C. Laboratory evaluation of the patient with erectile dysfunction. Endocrine 2004;23(23): 113-117

5. Baldo O, Earlley I. Diagnosis and investigation of men with erectile disfunction. Practicing Medicine 2005;2(1): 79-88.

6. Buvat J, Lemaire A. Endocrine Screenig in 1.002 men with erectile dysfunction: Clinical significance and cost effective strategie. J Urol 1997;158(5):1764-1767.

7. Johnson A, Jarow J. Is routine endocrine testing of ompotent men necessary?. J Urol 1992;147(6):1542-1543.

8. Korenman SG. New inshhights into erectile dysfuntion: a practical approach. Am J Med 1998;105 (2):135-144.

9. Roumeguere T, Wespes E, Carpentier Y, Hoffmann P, Schulman CC. Erectile dysfunction in associated with a high prevalence of hyperlipidaemia and coronary heart disease risk. Eur Urol 2003; 44:355-359.

10. Saltzam E, Guay A, Jacobson J. Improvement in erectile dysfunction in men with organic erectile dysfunction by correction of elevated cholesterol levels: A clinical observation. J. Urol 2004;172(1):255-258.
11. Rao K, Du GH y Yang WM. Correlation between abnormal serum lipid and erectile dysfunction. Zhonghua Nan Ke Xue. 2005;11(2):112-115.

12. Faiman C. Endocrine causes of impotence.. Cleve Clin J Med 1993; 60 (6):428-9.

13. Romeo JH, Seftel A, Madhon Z, Aron DC. Sexual function in men with diabetes type 2: association with glucemia control. J Urol 200;163(3):788-791.

14. Rholfing C, Little R, Wiedmeyer HM, England JD, Madsen $\mathrm{R}$, Harris MI et al. Use of GHb (HbAlc) in screening for undiagnosed diabetes in US population. Diabetes care. 2000;23:187-191.

15. Vela Navarrete R, García Cardoso JV, Cabrera Pérez J, González Enguita C, Casado Prez S, Farre J. Impotencia y enfermedad cardiovascular: Una nueva perspectiva en el compromiso asistencial del urólogo. Arch Esp Urol 2005; 58(1):43-53.

16. Seong DH, Ryu JK, Song S, Yoon SM, Suh JK. Elevated plasma transforming growth factor b1 level in patients with vasculogenic erectile dysfunction. Eur Urol Sup 2004;3(2):31

17. Ignarro LJ, Bush PA, Buga GM, Wood KS, Fukuto FM, Rasfer J. Nitric oxide and cyclic GMP formation upon electrical field stimulation cause relaxation of corpus cavernosum smoth muscle. Biocem Biophys Res Comm 1990; 170,843-850

18. Garcĺa Cardoso JV. Determinación del sistema óxido nítrico/GMPc en células mononucleares obtenidas de pacientes con disfunción eréctil. Tesis Doctoral. Universidad Autónoma de Madrid. 2004.

19. Block W, Klozt T, Sedlaczek P, Zumbe J, Engelmann U, Addicks $\mathrm{K}$ "Evidence for the involvement of endothelial nitric oxide synthase from smooth muscle cells in the erectil function of the human corpus cavernosum. Urol Res 1998;26(2):129-135.

20. Stanarius A, Uckert S, Machtens S, Stief C, Wolf G, Jonas U. Immunocytochemical distribution of nitric oxide synthase in the human corpus cavernosum: an ekectron microscopical study using the tyramide signal amplification technique. Urol Res 2001;29,168-172.

21. Kirby G, Jackson G, Betteridge J, Friedli K. Is erestile dysfunction a marker for cardiovascular disease?. Int $\mathrm{J}$ Clin Pract 2001;55:614-818.

22. López Farre A, Sánchez de Miguel L, Caramelo C, et al. Role of nitric oxide in autocrine control of growth and apoptosis of endothelialcells. Am J Physiol. 1997;272:H 760-H768.

23. De Frutos T., Sánchez de Miguel L., Farre J et al. Expression of an endothelial-type nitric oxide synthase isoform in human neutrophils: modification by tumor necrosis factor -alpha and during acute myocardial infarction" J Am Coll Cardiol 2001;37,800-807.

24. García Cardoso JV, García Méndez A, Mateos Cáceres P, Calahorra Fernández F, Lopez Farre A, Vela Navarrete R. Disfunción endotelial y disfunción eréctil: Un nuevo concepto con implicaciones diagnósticas y terapéuticas. Act Urol Esp Supp 2004;27(5):32.

25. García-Moll X. Marcadores de riesgo y pronóstico de los síndromes coronarios agudos. Cardiovascular Risk factors 2004; 12(6):352-364.

26. Kuller LH, Eichner JE, Orchard TJ, Grandits GA, McCallum L, Tracy RF. The relation betwwen serum albumin levels and risks of coronary heart disease in the multiple risks factor intervention trial. Am J Epidemiol. 1991; 134:1266-1277. 
27. Ridker PM, Cushman M, Stampfer MJ, Russel PT, Hennekens $\mathrm{CH}$. Inflammation, aspirin and the risk of cardiovascular disease in apparently healthy men. N Engl J Med 1997;336:973-979.

28. Libby P, Sukhova G, Lee RT, Galis ZS. Cytoquines regulate vascular functions related to stability of artherioesclerotic plaque. J Cardiovasc Pharmacol 1995;25(Suppl2), S9-12.

29. REAPE TJ, GROOT PH. Chemoquines and atherosclerosis Atheroesclerosis 1999;147:235-255.

30. Tatzber F, Rabl H, Koriska K et al."Elevated serum neopterin levels in atherosclerosis. Atherosclerosis 1991;89:203 208.

31. García Moll X, Cole D, Zouridakis E, Kaski JC. Increase serum neopterin: A marker of coronary artery disease activity in women. Heart. 2000;83:346-350.

32. Giugliano F, Esposito K, Di Palo C, et al. Erectile dysfunction associates with endothelial dysfunction and raised proinflamtory cytokine levels in obese men. J Endocrinol Invest 2004;27(7):665-669.
33. Dusitanod P, Eikelboom JW, Hankey GJ, et al. Homocysteine-lowering treatment with folic acid, cobalamin and pyridoxine does not reduce blood markers of inflammation, endothelial dysfunction or hipercoagulability in patients with previous transient ischemic attack or stroke: a randomized substudy of the VITATOPS trial. Stroke 2005;36(1):144-146.

34. Hankey GJ, Eikelboom JW, Ho WK, Van Bockxmeer FM. Clinical usefulness of plasma homocysteine in vascular disease" Med J Aust 2004;181(6):314-318.

Dr. J.V. García Cardoso

C/ Cocheras 4, Portal H, 3o 1

28007 Madrid

(Trabajo recibido el 24 de mayo 2005) 